\title{
Astrophysics Without Black Holes, and Without Extragalactic Gamma-Ray Bursts
}

\author{
Wolfgang Kundt ${ }^{1}$ \\ ${ }^{1}$ Argelander-Institut für Astronomie der Universität Bonn, Auf dem Hügel 71, D-53121 Bonn
}

Corresponding author: wkundt@astro.uni-bonn.de

\begin{abstract}
Contrary to common belief, it is argued that our Universe contains neither Black Holes, nor Extracalactic Gamma-Ray Bursts.
\end{abstract}

Keywords: black holes, gamma-ray bursts - galactic center - burning disks - magnetars.

\section{Introduction}

Modern Cosmology makes confident statements about processes which have happened throughout the Universe, all the way back to its beginnings, even though no convincing cosmological initial data have ever been reliably proposed [Hoyle et al 2000]. Such confidence is somehow based on the undoubted successes of Einstein's General Theory of Relativity in our immediate cosmic surroundings - the inner solar system - with or without a $\Lambda$-term which has been termed "dark energy" (by Mike Turner) when it has the sign opposite to that of energy, whose necessity is still debated. In these years, almost generally accepted as cosmic building blocks are (i) Supermassive Black Holes (SMBHs) in the centers of (almost all?) galaxies, without any physical justification, (ii) sources of almost all the GammaRay Bursts (GRBs) at cosmic distances, viz. of the bursts with line redshifts $\mathrm{z} \gtrsim 1$, and (iii) non-baryonic dark matter, even though we cannot even reliably measure the amount of ionised hydrogen between us and the (neighbouring) Andromeda galaxy [Braun 2013]..

In this presentation, I will restrict myself to the first two listed source classes, and start with the SMBHs. Not only have all the well-observed galactic central engines (CEs) defied a plausible $\mathrm{BH}$ interpretation, by their (large) luminosities, (high) temperatures, (strong) outflows, (relativistic) twin-jets, nonpointlike central potential, and halo-scale high-energy $\left(\lesssim 10^{2} \mathrm{GeV}\right)$ plumes, but even their theoretical existence is no longer justified because rigorous calculations have (recently) shown that the expectations of the 1970s have been hasty, that Roger Penrose's hypothesis of 'cosmic censorship' has been premature. We now know that complete gravitational collapse leads to naked singularities - not to BHs - except for a highest-symmetry subset of initial data, of measure zero. On top of not having been verified throughout more than 35 years, therefore, BHs are now no longer expected by theorists [Joshi 2009, Quevedo 2011, Joshi \& Malafarina 2013]. In particular, our Galactic Center (Sgr A*) deviates from containing a $\mathrm{SMBH}$ for at least 15 reasons [Kundt 2012], the 15th being the happy survival, so far, of cloudlet G2 during its ongoing approach of Sgr A*.

The second goal of this presentation is to refine my earlier considerations - dating back to the 1980s, when Galactic GRBs used to be common wisdom - that all detected GRBs come from local-Galactic neutron stars, rather than from ill-understood supersources at cosmic distances. Not only have there five quantitative distance estimates been published in the past which must not be forgotten, based on well-measured properties, which place their sources nearer than a few kpc from us, but meanwhile, a number of bursters have even been directly identified, and their tentative distances lowered, with the convergent result that we deal with the spectacular class of nearby magnetars in our Galaxy [Kundt 2008, 2010-2013, Ghisellini 2010, Archibald et al 2013, Rea et al 2013, Ho 2013]. The GRBs are a local-Galactic phenomenon, is my iterated message.

\section{BH Physics Reconsidered}

Black Holes have been baptised in Princeton, in 1971, by John Wheeler and Remo Ruffini. Their name stuck immediately, because years of hard international research had been invested, mainly in the United States and in Europe, until the complete family o f spinning BH spacetimes was finally published by Roy Kerr in 1963, and until the Holes' hairlessness had been (essentially) proven, during the decade to follow, with Brandon Carter among the cheerleaders. Mass, spin, and 
electric charge are a BH's (three) only free parameters [Heusler 1996].

But how to detect BHs in the Universe? Could we ever hope to identify a BH in the sky? Wouldn't they hide forever, on grounds of their expected dimness, their not being able to radiate? To my knowledge, this question was first asked seriously in 1972, during international meetings between theorists and observers, with a distinct emphasis on candidates of stellar mass. A first, promising suggestion was the X-ray emitting stellar binary system Cyg X-1 - the brightest stellar X-ray source in the Cygnus region - whose optically invisible component had to be more massive than a neutron star: bingo! Slowly, please: cannot the dark component be a neutron star surrounded by a self-gravitating disk [Kundt 1979]? Aren't self-gravitating disks stable? Disks are thought to form stars, their planets, and probably likewise all their moons; from what other instabilities could a disk suffer? Probably none. And how to blow the jets seen to spring forth from the dark component of the Cyg X-1-system? Binary neutron stars are observed to blow jets, whereas BHs cannot do that because they lack an inclined, co-rotating magnetosphere, for generating the jets' pair plasma [Kundt 2011]. In 1989, Daniel Fischer and I could not find a single BH in the whole class of (stellar-mass) BHCandidates [Kundt \& Fischer 1989].

In order to still detect a $\mathrm{BH}$ in the sky, should we perhaps search the Hole in a higher weight class, as a supermassive $\mathrm{BH}$, at the center of some galaxy, perhaps even our own? But how should it form there in the first place, or how else should it get to a galaxy's center? Are not self-gravitating disks stabilised radially by centrifugal forces, and vertically by pressure forces, if necessary via nuclear burning, like stars? I received "Honorable Mention" for this idea in 1978, by GRG, and described its structure more in detail at Bad Honnef in 1995 [Kundt 1996], in vital contact with Peter Scheuer, (see his contribution to that same book). From Suzy CollinSouffrin I had learned that mass spiral-in rates through galactic disks - determined indirectly by the central disk's luminosity - tend to balance mass-ejection rates through the Broad Line Region (BLR), determined by its emission-line intensities, whereby the ejection energy is supplied by nuclear burning, like in stellar explosions. Instead of like a $\mathrm{BH}$, the stable galactic disk would act like a Burning Disk, a "BD". Mass would spiral in through the disk, at a rate of $\lesssim \mathrm{M}_{\odot} / \mathrm{yr}$ for a galaxy like ours, and would subsequently be ejected explosively through its BLR, NLR, ELR out to the EER beyond the galaxy's halo, in the form of a galactic-scale fountain [Kundt 1987, 1990, 1996, 2005], cf. fig. 1, and [Kormendy 2011].

The case of our Milky-Way galaxy was my topic last year at Vulcano [Kundt 2012]; it will not be repeated here. No BH is needed for this fountain to function; on the contrary, a BH near the center would have prevented its formation, via irreversible accretion. It would have swallowed the incoming nuclear fuel, and thereby grown in mass. Instead, the SDSS plot of galacticcenter masses has shown a (statistical) monotonic decrease of CE masses from some initial $10^{9.3} \mathrm{M}_{\odot}$ down to the present-day rather low values, of order $10^{-3}$ that much, in the redshift range from $\mathrm{z}=4.5$ down to (the present) $\mathrm{z}=0$. Galactic CEs have monotonicly lost mass during cosmic evolution, defying a $\mathrm{BH}$ interpretation, see fig. 2 .

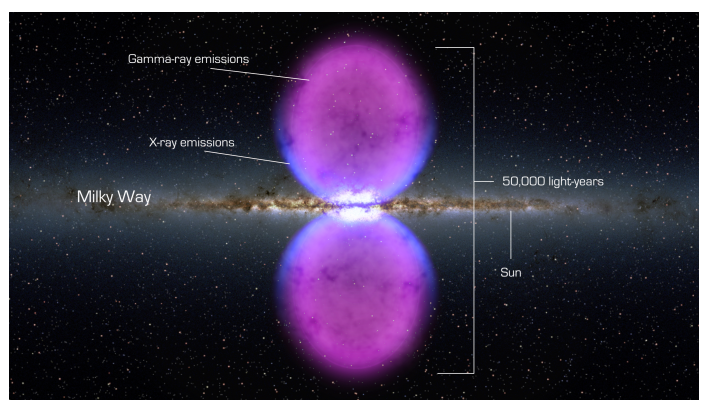

Figure 1: Most impressive evidence against a $\mathrm{BH}$ at our Galactic center: The FERMI Bubbles (or plumes), at photon energies $\lesssim 10^{2} \mathrm{GeV}$, probably emitted by buoyantly rising relativistic pair plasma from the near vicinity of Sgr A*, throughout the Milky-Way halo, to heights well above $20 \mathrm{kpc}$, taken from [Su et al 2010, 2011]. These same halo structures had already been detected and mapped decades earlier by [Sofue 2000], from radio and X-ray data.

At this point, my reasoning departs drastically from that of most of my colleagues, who use words like "downsizing" and "co-evolution" to describe the average monotonic shrinkage of CE masses plotted in fig. 2. They speak of a high BH 'efficiency', without indicating how a BH should do that, because as already stated in the Introduction, galactic centers are often observed to be quite luminous, stormy, jet-blowing, and pluming (at $\lesssim 10^{2} \mathrm{GeV}$ ), from their center all the way out into their halo. How is this central activity powered? My answer reads: by nuclear burning of the central disk, combined with magnetic reconnections in its (very) fast and differentially rotating corona [Kundt 1996]. A BD avails of abundant rotational, infall, and nuclear energy, for both non-thermal and thermal ejections: of radiation, jets, winds, and plumes. A SMBH would suppress all this.

Instead, a few of my decades-long friends call a $\mathrm{SMBH}$ the 'only thinkable' central engine (CE) of galactic centers without telling the reader how such a SMBH could have formed and grown, how it could e.g. emit the observed hard spectra [Aharonian et al 2004], and 
how it could perform the multiple hard ejections $[\mathrm{Su}$ et al 2010]. I think in particular of Max Camenzind, of Harald Lesch, and of Andreas Müller. They have published books, or contributed to books in which they claim, already on the book's cover, that mankind would not have formed without BHs [Müller 2010]. These publications have appeared fairly recently so that I have not gotten a chance to sort myself out with their authors, in friendly, animated discussions. How did my friends reach their present opinions? Have they found flaws in my 1996 model? Why did they never tell me?

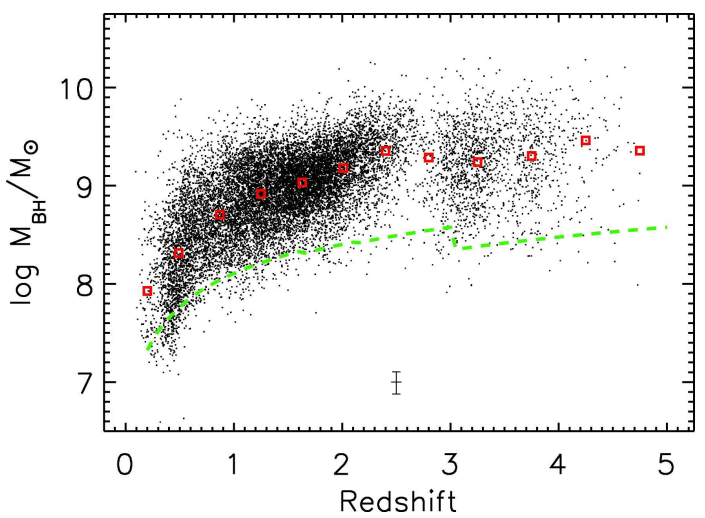

Figure 2: The SDSS plot of the CE masses $M(z)$ of all the $\left(\gtrsim 10^{4}\right)$ sampled galaxies by the Sloan Digital Sky Survey. as functions of cosmicredshift $\mathrm{z}(\geq 0.2)$, [Vestergaard et al, 2008]. Small squares denote (vertical) averages at fixed redshift bin. The plot shows that $\mathrm{M}(\mathrm{z})$ decreases monotonicly, from $10^{9.3} \mathrm{M}_{\odot}$ at $\mathrm{z}=4.5$ down to $\lesssim 10^{8} \mathrm{M}_{\odot}$ at the smallest plotted redshift, $\mathrm{z}=$ 0.2 .

The scientific situation is, in fact, more inconsistent than described so far. Exact analytical calculations have meanwhile shown that mass concentrations collapsing under their own weight will no longer form BHs, rather naked singularities, except for configurations of highest symmetry which are, however, of measure zero among all initial data [Joshi 2009, Quevedo 2011, Joshi \& Malafarina 2013]. Penrose's hypothesis of "cosmic censorship" ought to be strengthened into the hypothesis of an "avoidance of unhalted gravitational collapse", AUC, unless we do not mind contenting ourselves with a piecewise singular theory, or unless we are ready to search for a replacement of GR by some new, nonsingular spacetime theory. My own preference is for AUC, as I have never failed to find hurdles to any proposed unhalted gravitational collapse.

\section{Gamma-Ray Bursts from Galactic Magnetars}

Now to item (ii) of this pamphlet: What are the sources of the GRBs? Isn't it clear since some 20 years that those sources 'must be' at cosmological distances? To my mind, it has never been: True: the bursts reach us from all directions, three per day on average from outer space, in an almost isotropic fashion, but small anisotropies of well-defined subclasses of bursts have repeatedly been published; the isotropy is not perfect, and a nearby population of sources $(\lesssim 0.3 \mathrm{kpc})$ can certainly not be excluded, in particular when individual bursters have anisotropic radiation patterns, oriented with respect to their (low-mass) accretion disks whose normals prefer to lie in the Galactic plane. (Such a preference is expected for disks assembled by neutron stars during their oscillatory motion through the Galactic disk).

Next think of the redshifts $\mathrm{z}$ of the bursts' afterglows, and host galaxies, z lying between 0 and 9, corresponding to Lorentz-factors $\gamma$ between 1 and 5 , according to the kinematic law $\mathrm{z}+1=\gamma(1+\beta)$. Such redshifts may well be kinematic redshifts of matter ejected centrifugally from near the speed-of-light cylinder of a sufficiently magnetised, slowly spinning neutron star, hence need not mean a (cosmologically) large distance. Note that a few bursts have even had $\mathrm{z}=0$, including those from the SGRs, so that a distal interpretation of $\mathrm{z}$ would deal with a range of distances through a factor of order $10^{8}$, corresponding to a range of powers through a factor of $10^{16}$, for essentially the same type of sources, viz. accreting neutron stars. This reason alone has never encouraged me to take a cosmic interpretation of the GRBs seriously: The distant sources would have to radiate almost infinitely times brighter than their cousins in the Milky Way, even if you try to save a factor of $\gamma^{2} \lesssim 10^{6}$ by postulated beaming (of both the prompt and the afterglow emission!). To me, the redshifts mean velocities, not distances (of the sources). Note that there is no problem with missing blueshifts from spherical explosions: Only the first flash from a distant, spherical explosion is blueshifted, all later radiation (from a small angular neighbourhood of the explosion site, due to scattering) is received redshifted, mainly from the receding polar cap of the expanding sphere of ejecta.

And what about host galaxies, at the same redshift? Only a quarter of all bursts have had reliable-looking hosts, whereby no host galaxy has ever been intrinsicly brighter than the LMC! Fancy the gigantic intrinsic energy $\left(\gtrsim 10^{53} \mathrm{erg}\right.$ ) of a GRB interpreted cosmologically, illuminating the CSM of its host galaxy: The host would have to flare, rather than be just about detectable. No 'ordinary' galaxy (like ours) has ever hosted a GRB! .My preferred explanation: What observers call 'host' are the flaring ejecta plus dragged-along CSM of the (nearby) bursting magnetar, before the hadronic ejecta start losing a significant fraction of their kinetic energy received at launch. We receive cooling radiation from 
thermalised (kinetic) burst energy, around the magnetar, part of which is still moving transrelativistically away from us, with the same redshift as the early ejecta. All bursts come from inside our Milky Way; the published 'hosts' are all fake.

Now let us look again at the five published distance estimates for GRBs, all of which imply source distances of less than a few kpc, depending on the strength S of the recorded burst [Kundt 2010]. The first three of them take similar shapes:

$$
d / k p c<\left\{1 / \sqrt{S_{-4}}, \quad \gamma \sqrt{L_{38}}, 1 / \sqrt{S_{-11}}\right\},
$$

in which $\mathrm{S}$ stands respectively for the peak incoming energy-flux density of the prompt at $\gtrsim \mathrm{MeV}$, early optical afterglow $\}$ emission in the \{first, third\} entry, where $\mathrm{S}_{-4}:=\mathrm{S} / 10^{-4} \mathrm{erg} / \mathrm{cm}^{2} \mathrm{~S}$ is evaluated in cgs units (as always), and $\gamma$ stands for the (assumed) bulk Lorentz factor of the neutron-star system w.r.t. which the power $\mathrm{L}$ is emitted.

More in detail, the first estimate in (1) uses photon crowding at the source, which would soften a GRB's spectrum above the pair-formation threshold (of $\approx \mathrm{MeV}$ ) [Schmidt 1978, Baring 1992] unless it were emitted in a highly beamed way. It rules out a distance of $50 \mathrm{kpc}$ (of LMC) for the SGR 0526-66 - which flared on 5 March 1979 - and it likewise rules out a distance of $8.7 \mathrm{kpc}$ for SGR 1806-20; in the Main Table of McGill SGR/AXP Online Catalog. In the meantime, the FERMI mission has measured energetic hard tails to many GRBs reaching up in energy towards $\gtrsim 10 \mathrm{GeV}$, which begin to reach us right after the prompt emission, see [Ghisellini 2010], and Eleonora Troja's contribution to this workshop. Even so, their roof-like spectral peak (near $1 \mathrm{MeV}$ ), at the top of the Band spectrum, did not look grossly distorted in the new spectra. For all of them, therefore, distances larger than one kpc are inconsistent with their (hard) spectra unless their power was emitted in a highly beamed fashion, whose detailed mechanism has never been explained convincingly at conferences, or anywhere in the literature. Required would be hadronic jets formed on the timescale of seconds, but lasting long enough (months) in order to allow likewise for beamed afterglows. Such postulated jets would have to function very differently from the hundreds of well-studied quasi-stationary pair-plasma jets observed to spring forth from the various well-known jet sources, all powered by heavy rotating magnets [Kundt 2011].

The second inequality in (1) holds under the assumption that the source of the GRB is a neutron star, whose small surface area restricts its maximal possible luminosity L, via the Eddington constraint. It was always satisfied during the 1980 s, when scientists trusted the Galactic-neutron-star model for the GRBs.
Finally, the third estimate in (1) assumes that the afterglows are emitted incoherently, from the near surroundings of the burst sites which are hit by the explosion. Bright afterglows tend to have Poynting fluxes $\lesssim 10^{-10} \mathrm{erg} / \mathrm{cm}^{2} \mathrm{~s}$, for which (1) yields distances $\mathrm{d} \lesssim 0.3$ kpc., in accord with the estimated magnetar densities in the Milky Way. Again, this third estimate would not hold for jetlike emission; but how, or why would such be expected?

Two even much smaller distance estimates d have been derived for the nearest among all GRBs, for which we even see their much more frequent but much fainter repetitions, for the Soft Gamma-ray Repeaters (SGRs), of which 13 are presently listed in the McGill Catalog. (Only 8 SGRs were listed in [Kundt 2010]). So far, none of them has flared more than once at their large, original intensity, but their intense flares were much brighter than all those of the non-repeaters. For this reason, I feel encouraged to use again the second estimate of (1), but this time not with the classical Eddington luminosity $\mathrm{L}=10^{38} \mathrm{erg} / \mathrm{s}$ of a steadily accreting neutron star, but instead with the maximal possible luminosity expected for an isolated, clumpy impact onto a neutron star, of order $\mathrm{L}=10^{42} \mathrm{erg} / \mathrm{s}$. Another, independent estimate involves the typical Galactic peculiar velocity c $\beta$ of a magnetar (blowing radio bubbles, with measured angular velocities), of order $\beta \lesssim 10^{-3}$. The two estimates read [Kundt 2010]:

$$
d / 30 p c \lesssim\left\{\sqrt{L_{41.9}}, \quad \beta_{-2.9}\right\},
$$

in agreement with the nearest expected magnetar distances, and also with our additional knowledge about the SGRs. All these distance estimates are some $10^{8}$ times smaller than their cosmological counterparts, given by $\mathrm{d} \approx \mathrm{z} \mathrm{c} / \mathrm{H}_{0}$.

So where have we arrived? In the first three paragraphs of this section, we have argued that neither quasi-isotropic arrivals, nor relativistic redshifts $(\mathrm{z} \lesssim$ 9 ), nor frequent (seeming) host galaxies needed imply cosmological source distances for plausible source properties. Then, in the following five paragraphs, we have repeated earlier inequalities, all of which rule out extragalactic distances for magnetar models. In the future, it will certainly help to get more reliable distance estimates for the small subset of already identified burst sources.

But let us look once more at the evidences: To begin with, all the soft repeaters have been directly identified, as GRBers, during their first, large bursts, with measured spin rates during their early afterglow oscillations. Unfortunately, their distances are highly controversial, with GRB 050379 as their worst case, projecting onto N49 in the LMC, though having been some $10^{6}$-times too bright during outburst for that (large) distance, in- 
Astrophysics Without Black Holes, and Without Extragalactic Gamma-Ray Bursts

consistent with its Band spectrum [Schmidt 1978, Baring 1992]. Next the AXPs: They share the (long) spin periods of the SGRs, qualifying them as dead pulsars, after $10^{6.4} \mathrm{yr}$ of active pulsar spindown [Kundt 2008]. Their 'anomaly' as permanent X-ray emitters can be understood as their being surrounded by low-mass fallback disks, from the collapsed cavity blown into their CSM during their past pulsar activity. Their (comparatively large) number agrees with that of the dying pulsars, and with their expected small distances from us, inconsistent with certain estimates in the literature.

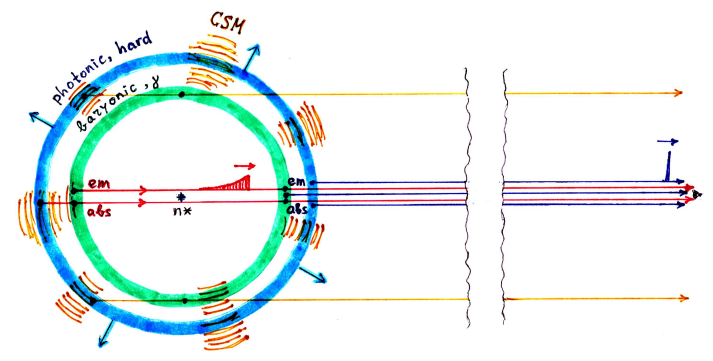

Figure 3: Schematic drawing of the expected ejection geometry of a GRB by a magnetar: An ultramassive clump from the inner edge of its (low-mass) accretion disk hits the surface of the central neutron star, heats up, expands, and gets seized and centrifugally re-ejected by the magnetar's strong, corotating magnetosphere, near its speed-of-light distance, with Lorentz factors $\gamma \lesssim 5$. As a result, a hard electromagnetic flash is emitted (mostly peaking near $1 \mathrm{MeV}$, but ranging from radio frequencies up through IR, optical, X-ray, and $\gamma$-ray frequencies all the way towards and beyond $10^{2} \mathrm{GeV}$ photon energies), closely followed by a centrifugally ejected transrelativistic hadronic shell. This couple of two more or less radially emitted and/or boosted transrelativistic shells of ejecta impact on the GRB's CSM, they both heat up, and give rise to the burst's prompt and afterglow emissions, as well as to the evolving appearance of a 'host galaxy'. This model can be shown to be flexible enough to account for all the observed GRBs, including their intrinsic differences, and occasional anomalies, such as short and long bursts (due to repeated accretions), pre-cursors and post-cursors (for the same reason), redshifts, early afterglow oscillations (by the output of the spinning central neutron stars), strong flaring of the X-ray afterglows (during collisions with the CSM), linear polarisations (for scattered radiation), and so on and so forth.

Independently of the distance problem, the hard spectra of the GRBs - reaching up to and beyond $10^{2} \mathrm{GeV}$ - tell me that their sources involve neutron stars. These neutron stars must not be isolated, or else they would be radio pulsars. Which means that we deal with strongly magnetised neutron stars, though not in excess of $10^{14} \mathrm{G}$ surface strength, a neutron star's extreme field [Kundt 2008]. The bursters' occasional strong magnetic torques can be achieved via confinement by a surrounding low-mass accretion disk, which explains their name 'magnetars'. Their (invisible) disk was first indicated by glitches of the SGRs $1900+14$ and 1806-20, during which their spindown rates increased, i.e. required a stronger torque after the glitch, and has been recently fostered by the anti-glitch of the AXP $1 \mathrm{E}$ 2259+586 [Archibald et al 2013], whose sudden spindown cannot be understood (to my mind) without a strong centrifugal mass ejection [Kundt 2010].

And how do the magnetars emit their bright, hard bursts? Fig. 3 sketches my understanding of how they are thought to do it. Every now and then, at large temporal separations (of $\gtrsim 50 \mathrm{yr}$ ), a supermassive clump $\left(\gtrsim 10^{16} \mathrm{~g}\right)$ accretes onto a magnetar's surface, from the inner edge of its surrounding accretion disk, via Rayleigh-Taylor instabilities. Pre- and post-cursors occur when even several successive such clumps fall down. The freefall potential of a heavy clump, crashing onto a neutron star's surface, creates transient temperatures near $10^{12} \mathrm{~K}$ on impact, so that matter gets ionised, and raised to scale heights $\mathrm{kT} / \mathrm{mg} \gtrsim 10^{6} \mathrm{~cm}$.. This lifted, ionised matter is seized by the $\mathrm{n}^{*}$ 's corotating magnetosphere, and centrifugally ejected at speed-of-light lever arms, with Lorentz factors $\gamma \lesssim 5$. This hot re-ejected matter emits a bright photonic flash, closely followed by an intense, transrelativistic hadronic flash. When these two successive outgoing flashes hit the burster's CSM, they cause its afterglow emission, from radio frequencies up to $10^{2} \mathrm{GeV}$ energies, and eventually also the burst's seeming 'host galaxy'.

\section{Summary}

Old and new arguments have been compiled that BHs are no longer even plausible, and that all GRBs are emitted by the Galactic magnetars.

\section{Acknowledgement}

My warm thanks go to Ole Marggraf for help with the proceedings, and to Thomas Tauris for acquainting me with up-to-date literature on magnetars.

\section{References}

[1] Aharonian, F., et al (100 authors): Astron. Astrophys. 425, L13-L17, 2004.

[2] Archibald, R.F., Kaspi. V.M., et al (9 authors): An anti-glitch in a magnetar, Nature 497, 591-593, 2013. doi:10.1038/nature12159 
[3] Baring, M.G.: Spectral breaks for theorists, Nature 358, 624, 1992.

[4] Braun, R.: The cosmic web in focus, Nature 497, 191-192, 2013. doi:10.1038/497191a

[5] Ghisellini, G., Ghirlanda, G., Nava, L., Celotti, A.: $\mathrm{GeV}$ emission from GRBs: a radiative fireball?, MNRAS 403, 926-937, 2010. doi:10.1111/j.1365-2966.2009.16171.x

[6] Heusler, M.: Black Hole Uniqueness Theorems, Cambridge Lecture Notes in Physics 6, 1-249,1996.

[7] Ho, Wynn C.G.: More than meets the eye: magnetars in disguise, MNRAS 429, 113-118, 2013.

[8] Hoyle, F., Burbidge, G., Narlikar, J.: A Different Approach to Cosmology, Cambridge Univ. Press, 2000.

[9] Joshi, P.S.: Sci. Am., February, 26-33, 2009.

[10] Joshi, P.S., Malafarina, D., Instability of BH formation under small pressure perturbations, in: Gen. Rel. Grav. 45, 305-317, 2013.

[11] Kormendy, J., Bender, R. : Nature 469, 374-379, 2011.

[12] Kundt, W.: Cygnus X-1 - a Neutron Star Surrounded by a Massive Disk? Astron. Astrophys. 80, L7-L10, 1979.

[13] Kundt, W.: Our Galaxy - a former Seyfert?, Astrophys. \& Space Sci. 129, 195-201, 1987.

[14] Kundt, W.: in: Jets from Stars and Galactic Nuclei, Lecture Notes in Physics 471, 265-270, 1996. doi:10.1007/BFb0102617

[15] Kundt, W.: Astrophysics, A New Approach, Springer, 147-152, 2005.

[16] Kundt, W.: Pulsar Physics without Magnetars, Chin. J. Astron. Astrophys. Vol. 8, Supplement, 213-218 (2008).

[17] Kundt, W.: The Local-Galactic interpretation of the Gamma-Ray Bursts, in: Multifrequency Behaviour of High-Energy Cosmic Sources, MemSAIt Vol. 81, N.1, 449-459, 2010.

[18] Kundt, W.: Black Holes cannot blow Jets, in: XIV $^{\text {th }}$ Brazilian School of Cosmology and Gravitation, Mangaratiba 2010, eds. Novello \& Bergliaffa, Cambridge Sci. Pub., pp.109-119, 2011.

[19] Kundt, W.: Our Galactic Center - the nearest Burning Disk, FRASCATI WORKSHOP 2012, overdue.
[20] Kundt, W.: The Inorganic Machines of the Universe, in: $\mathrm{XV}^{\text {th }}$ Brazilian School of Cosmology and Gravitation, Mangaratiba 2010, eds. Novello \& Bergliaffa, Cambridge Sci. Pub., $\geq 2013$.

[21] Kundt, W., Fischer, D.: Is there a Black Hole among the BHCs?, J. Astrophys. Astron. 10, 119$138,1989$.

[22] Müller, A., Schwarze Löcher, Die dunklen Fallen der Raumzeit, Spektrum Akademischer Verlag Heidelberg, 1-206, 2010.

[23] Quevedo, H., Multipolar Solutions, in: XIVth Brazilian School of Cosmology and Gravitation, eds. Novello \& Bergliaffa, Cambridge Sci. Pub., 83.97, 2011.

[24] Rea, N., Pons, J., Torres, D., Turolla, R.: The Fundamental Plane for Radio Magnetars, ApJ. 748, L12 (6pp.), 2012.

[25] Schmidt; W.: Distance limit for a class of model GRB sources, Nature 271, 525-527, 1978.

[26] Sofue, Y.: Bipolar Hpershell Galactic Center Starburst Model: Further Evidence from ROSAT data and X-ray Simulations, ApJ 540, 224-235, 2000.

[27] Su, M., Slatyer, T.R., Finkbeiner, D.P.: Giant Gamma-ray Bubbles from Fermi-LAT: AGN activity or Bipolar Galactic Wind?, ApJ 724, 10441082, 2010.

[28] Su, M., Slatyer, T.R., Finkbeiner, D.P.: FERMI Bubbles: A $10 \mathrm{kpc}$ Shock from the Galactic Center, $A A S$ 43, 2011.

[29] Vestergaard, M., et al (5 authors): Mass functions of the active BHs in distant quasars from the Sloan Digital Sky Survey Data Release 3, ApJ 674, L1L4, 2008.

\section{DISCUSSION}

PIETER MEINTJES: You propose an extended burning disc, instead of a supermassive $\mathrm{BH}$ in the center of the MW galaxy. What is the size of that disc in relation to the orbits of the innermost stars that orbit that region? Also, what influence will such an extended disc have on the orbits of these stars compared to a $\mathrm{BH}$, for example?

WOLFGANG KUNDT: Thank you, Pieter, for asking me these constructive questions. In my approximation, the $\mathrm{BD}$ inside $\mathrm{Sgr} \mathrm{A}^{*}-$ the $\mathrm{CE}$ of the MW galaxy - has a radius of $\approx 10^{14} \mathrm{~cm}$, whilst the peri-astron distance of the innermost monitored star, 
$\mathrm{S} 2$, measures $\gtrsim 10^{15} \mathrm{~cm}$. As already mentioned in my talk of last year [Kundt 2012], the orbit of S2 showed a peri-astron advance of $3 \mathrm{deg}$ after its first revolution, consistent with a MacLaurin mass distribution (instead of pointlike potential), and six successive measured po- sitions near peri-astron were offset by $\lesssim 10$ marcsec towards NE, which I interpret as a fata morgana in the infrared (caused by the high plasma density in the inner disk). At this same time, the star S2 flared by half a magnitude, plausibly due to friction on the disk. 